\title{
Model of the Different Heterogeneous Networked Database Systems at the Medium and Large Companies in Kosovo
}

\author{
Dr. Sci. Edmond Beqiri \\ University of Peja "Haxhi Zeka" \\ edmond.beqiriunhz.eu \\ Mr. Sci. Genc Beqiri \\ PhD Candidate - University of Skopje \\ gencgb@evun.eu
}

\section{Doi:10.5901/ajis.2015.v4n2p519}

\begin{abstract}
Modern companies use Local and Regional Networks, as well as the Internet, for everyday Business. With the latest developments in the region, this has become relatively easy, since companies have a full support to develop the IS by the ISP in a manner of: Centralized (Hierarchical) IS; Decentralized (Distributed) IS or Combined (both hierarchical and distributed) IS. Since the company branches and hence its resources lay within one single state, the network is usually provided by the same ISP. This means that the offer includes Internet, DNS, E-mail service, Intranet and VPN. Now, most of the services provided are designed to be used all over the WWW and the offer itself consists of very ingenious solutions. The development of applications for Internet / Intranet seeks to overcome the limitations of Web forms interface. The nature of work of Companies distributed around a state requires a combination of various types of communication means as: Wired Networks; Wireless Networks; Meshing Networks; GPRS Networks. In the case of bigger trading Companies, the number of processes that has to be managed, as well as very large number of various items-codes, in combination with the need to serve immediately, makes VPN Networks more attractive. VPN makes possible the use of: Local Data Servers; Local Application Servers; Distant Central Data Servers; Combination of Wire Networks with Wireless and GPRS Networks. The expansion of the business in other neighboring countries complicates the communication between branches and central. The need for immediate information stays the same, but, since we usually have more than one different ISP, the solutions becomes a little more complicated. This paper offers the original solution how the communication between branches has to be done using Bridges and VPN tunnels between the networks of two different ISP-s.
\end{abstract}

Keywords: Heterogeneous, Networks, ISP, IS, Wired, Wireless, Meshing, GPRS.

\section{Introduction}

Recently, last several years, Internet has become the global standard for communications. The Internet is a global network of computers working on a set of TCP / IP network protocol. World Wide Web (WWW), is one of the many services that the Internet works, it brought opportunities for access to documents in various formats.

Internet can practically be used on almost any type of computer or operating system.

Now, the Internet is the host of many companies and millions of Web sites of organizations that provide various types of services. The Internet is marked as a new model for systems developers because it provides multiple benefits, such as, universal access to data, common interface GUI, lower cost on preparation of end users, etc.

The development of applications for Internet / Intranet seeks to overcome the limitations of Web forms interface.

The administrators of databases in today's Internet-based environment have to understand the implications of the development of Web applications with data bases in terms of supporting the extended type of data security, transaction management, and designing the database.

\section{Regional Network (Internet) in SEE}

The Internet is constructed using virtually any type of environment broadcaster, such as:

- Fiber optic lines 
- Telephone lines and

- MRF - Microwave radio frequencies and channels.

- Wi- Fi technology

- GPRS

Since the need for TCP / IP addresses has increased, the network has started to migrate from standard protocol IPv4 (Internet Protocol Version 4) with 8 bit value (32-bit addresses), to the new IPv6 protocol - more advanced than IPv4, the standard 12 bit value (128-bit address), that increased the number of individual addresses to 4095 . Numbers 0 and 4095 (all bits set). This way we can increase the number of addresses is being applied on substantially.

\section{Databases and Internet Technologies}

The Internet provides universal access through global interfaces known as Web Browser, which is easy to use and works across multiple platforms. Connectivity of databases to the Web opens the doors of new innovative services such as:

- Allows immediate response to competitive pressure by bringing new services and products to the market quickly.

- Increase customer satisfaction through the creation of supportive services based on Web.

- provide rapid and effective delivery of information through universal access, from across the street and up to the other side of the globe.

Having all these advantages, many departments of information technology services have to deal with the need to create universal architectures for data access, based on Internet standards, to guide appropriate operations and to facilitate decision making.

\section{Company and Network organization within the Same Country and the Same ISP}

As we can see the Company that is lying within the same Country and has its network provided and managed the same ISP has some preferences because both in technical, organizational and lawful mode has the advantage of applying the same rules in all of its branches.

Now the companies organization depends on its stretch in the Region, number of units (branches), variety of its business, number of employees, and the technology used for business administration.

In the case of larger Trading Companies these are Crucial.

Since they are dependent on the service they give to the customer, their organisation must also reflect the way they need to do business.

Based on all this the organisation schema should be:

- Centralized (Hierarchical) IS;

- Decentralized (Distributed) IS or

- Combined (both hierarchical and distributed) IS.

We recommend the Combined (both hierarchical and distributed) IS for the Company, especially in the case of trading companies spread throughout the country.

The Wide Area Networks that we are using are of great importance and very helpful in organising our business, but, they are in the phase of spreading through the area and still have problems with uninterrupted operation.

Having the Combined organisation allows us to be independent on the wide are infrastructure.

We can organise business locally within our local Network, with occasional links to the Central Servers and databases.

This way we can ensure that our business is independent of the external (ISP) problems for a period of time, since we organise most of the doings to be executed locally, and from time to time ( periodically) update the Central database with the fresh and detailed information.

Central database is crucial for business progress because it enables the company to automatically refill the branches with items that are sold meantime and need to be supplied. Without it we would have an unorganised supply chain and it would bring to a situation where some branches shortage and the others have overstock.

The Network organization reflects these needs, and must be designed to fulfil these requirements.

This can be best achieved through a VPN (Virtual Private Networking) Network organisation. VPN is enabled making tunnels inside our Internet/Intranet Network layout. The tunnels connect the points needed directly with both TCP/IP and DNS infrastructure, ensuring the internal connection and organisation without exposing those points to the 
external view. For an external entry we need to allow a Public IP address through a connection to an internal IP address, a process named NAT (Network Address Translation), and it can only be performed by collaboration of Internal and ISP IT staff. This ensures the safety of our transactions.

VPN makes possible the use of:

- Local Data Servers;

- Local Application Servers;

- Distant Central Data Servers;

- Combination of Wire Networks with Wireless and GPRS Networks.

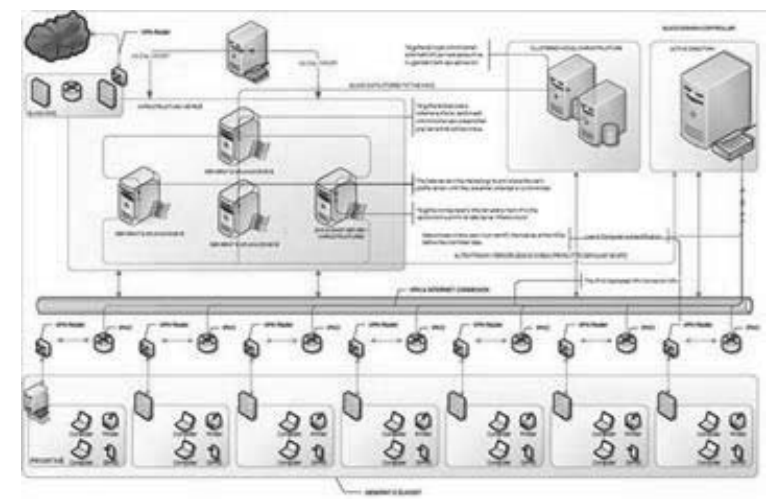

Figure 1. Overall Network scheme of the IS layout

\section{Database and Server Organization Based on the Network Layout}

The data processing system is both dependent on the Network layout, and in the same time it also imposes the way the Network is organised. We are going to use:

- Central Database (CDB) located on a Central Sever ( one or two positions)

- Application Servers for CDB access

- Local Main Databases (LMDB) located on Local Servers in the physical position of the Branches

- Application Servers for LMDB access

- Local Databases (LDB) placed on Local POS (Points of Sale)

- Local POS can have a

- Normal Database (NDB) for use of PCs or

- Compact Database (CDB) for use of Handheld computers or other SMART devices

Data Flow is bidirectional,

- from the CDB towards LDB-S and from LDB-S towards POS, which will reflect the change of any basic information needed, and

- From POS to LDB, then from LDB to CDB (or even directly from POS to CDB), transmitting the local turnover to the Server databases, both local and Central 


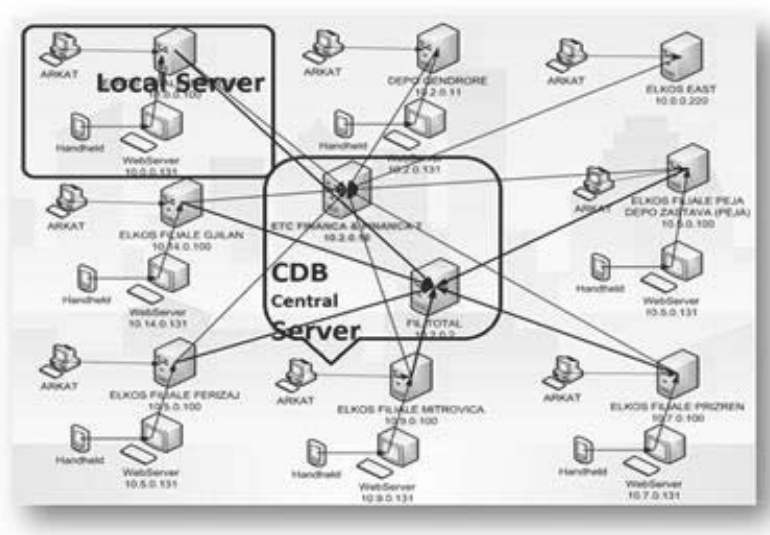

Figure 2. Global Network scheme of the IS layout

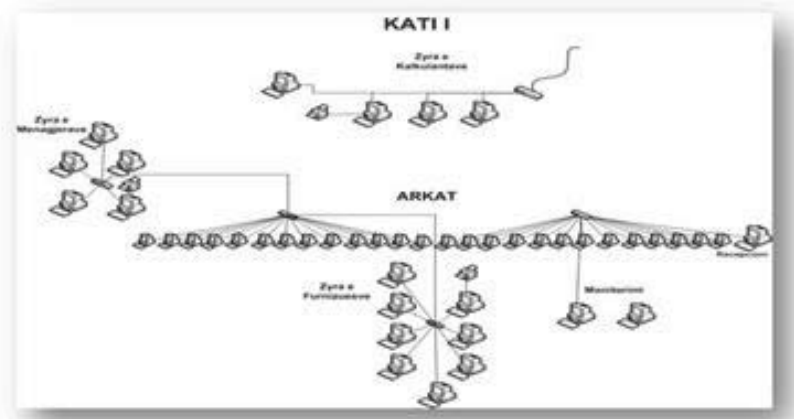

Figure 3. Local Network scheme of the Branch IS

The bidirectional flow will fulfil our Companies need for information on creating and using back office reports needed for further planning and developing.

Once we have obtained a database on a server in a data processing environment further successful processing depends on communication ability.

The means of data processing can be organised by:

- Client Server application or/and

- Web based Applications

One of the most useful is IIS (Internet Information Services) that comes as part of the Windows operating system. It allows various operations that require access to the Internet.

It is a software service that supports the creation of Web sites and other Internet functions:

- WEB interface

- Network News Transfer Protocol (NNTP)

- File Transfer Protocol (FTP)

- Simple Mail Transfer Protocol (SMTP).

The use of these services allows us to extend our Network throughout the region that we operate. This includes Internet and Intranet.

First the IIS has to be activated and set up on our Server 

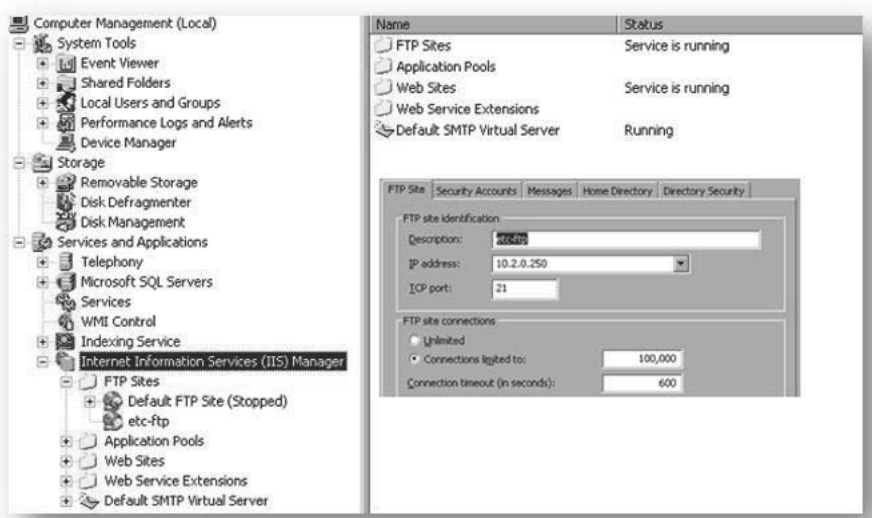

Figure 4. IIS settings

After setting up a Domain all the demands for server acces are directed through the service.

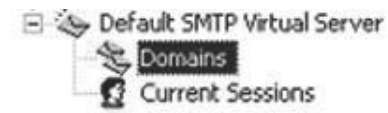

And finally Web site and Web service that will manipulate the data from the Internet / Intranet

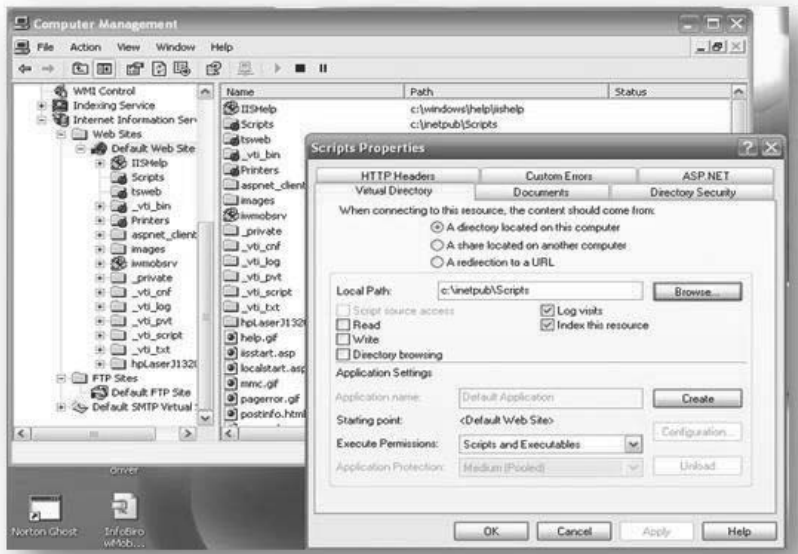

Figure 5. Web Site and Web service enabled

We have practically enabled Data Base access through the scripts residing on our server.

The procedure of searching and updating a commercial web site:

- Browser requests access to Web site Located on a Web Server Xx (Web Page)

- Xx has enabled Web server through which to connect to the database

- Server database gives access DBMS (in the case of SQL moment) 
- SQL processing application results are returned to the Web Server $x X$

- Xx Web Server Web Browser presents results

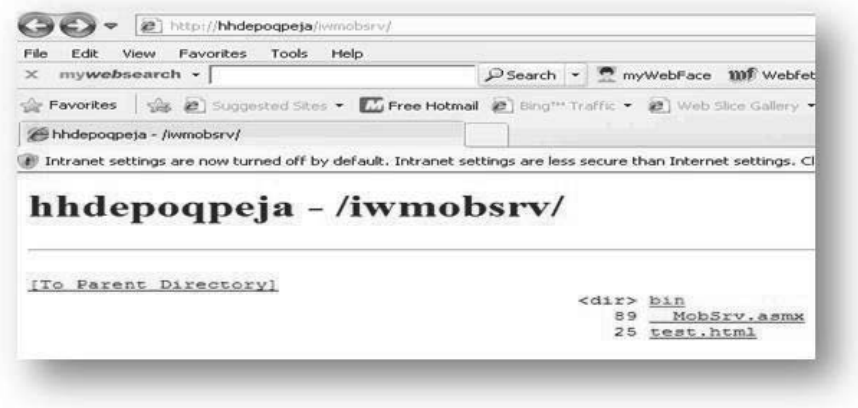

Figure 6. Scripts applied through the Web Site and Web service

The code used is in Visual Basic Script - VBScript.

We have practicaly used Network redirection

- From the starting Demand that can be on any kind of device

- To the Internet / Intranet through a WEB Service

- $\quad$ On the Server where we have SCRIPT-s residing

- Finaly getting access on the Data Base and retreiving or updating the information.

\section{Requirements towards Network Organization and Multiple ISP Coordination when Company Expands Outside the Country}

In the companies' further development, we have a new moment. The company is spreading its business outside the borders, towards other countries in the region. This brings new requirements towards Networking and Database processing. There are also legal matters to be considered but we are not going to deal with them at the moment.

Since we need to manage the new branch from within the central of the Company, we also need access to its Databases and other IS resources. This can be done by the means of Internet utility programs like: team Viewer, Logmein, remote connect ect., but they are for the occasional use and not suitable to work with them all the time.

The connection can be achieved by setting up a Public IP address, public domain and use an internal Network distributor to the internal LAN. This is not very comfortable and safe, and our data security is of outmost importance. The connection through a public address looks like:

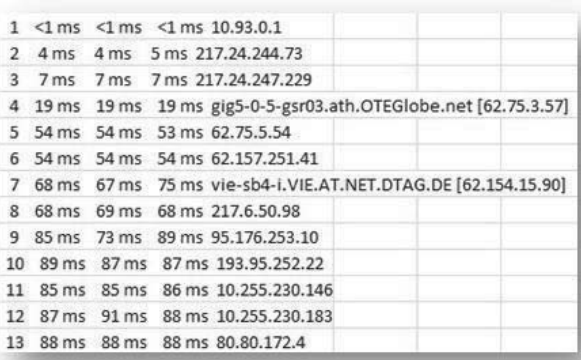

Figure 7. Number of hops needed through a public address

It can also be set through installation of VPN Network between two Networks. This gives us the means to enable data access to the users both ways with a higher degree of safety. 
The Pubic addresses are only used to achieve a PTP (point to point) connection. After that all levels of data security are integrated in the existing system.

The two network connection between two different networks is obtained by building an IPSec tunnel. Both ISP-s have to provide configuration parameters. The parameters are such as: Peer's IP address, Encryption Algorithm, Transform (IPSec protocol), source IP, and Port number or All.

Once we apply the parameters to the link we have attained the link. Now this Connection has only three hops to be achieved:

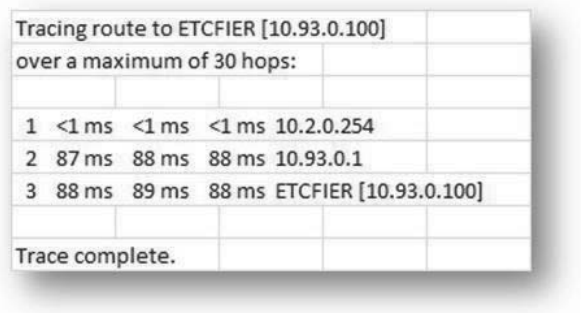

Figure 8. Number of hops needed through a VPN address

It is achieved through only 3 hops, which results in a faster network, it is functional and the local users are using all the resources as though they were locally attached. The abroad branch is working and we can treat it as if it were here.

The Local Database of the abroad Branch is directly connected to the Central Database of the Company and the flow of data and information is both ways. We can continue with work at real time connection, send and retrieve data.

\section{Conclusion}

Modern Companies work fast and have a need of a flow of information that exceeds local boundaries. This means that the Companies have to extend from Local to Regional and further to interstate business. This automatically calls for development of Local networks, Wide area networks, and to International Network (internet).

For a Company to put forward it needs to hire staff capable of enhancing business. For that staff to work freely we have to develop technologies that work for them. These technologies need an environment where they can work. They also need information and a fast and secure interchange of that information.

Same as the Companies expand the technology used has to communicate and to provide all means of information, preceding the development of the Company itself.

IT Systems and IT technology has a duty to provide information and communication between all parts of the Company wherever it is located.

To achieve all this, we need powerful Servers, meaningful Databases and fast and secure Networks that enable that flow of information.

Most of the time our partners who have taken a duty of providing for us, give us the solutions needed. Since they are also in the phase of development, sometimes we have to find the solution with their help.

In the above described, we tried to describe the solutions and the software, hardware and network needed for successful work and expansion of a Company inside one country and in the nearby Countries.

\section{References}

Abrams, B. NET Framework Standard Library Annotated Reference, Volume 2: Networking Library, Reflection Library, and XML Library, 2008.

C. Rapier and B. Bennett, "High speed bulk data transfer using the SSH protocol", MG '08: Proc. of 15th ACM Mardi Gras Conference. pp. 1-7, 2008.

C. Rapier, M. Stevens, Application Layer Network Window Management in the SSH Protocol , Presented at Supercomputing Conference 2004

Date, C.J. An Introduction to Database Systems, 8th Edition. Addison-Wesley, Reading MA, 2003.

Davidson, L. Ten Common Database Design Mistakes, http://www.simple-talk.com/sql/database-administration/ten-common-databasedesign-mistakes/, 2007. 
Mauring, F. Development and Transition. Available at: http://www.kosovo.undp.org/repository/docs/Dev_transit_eng.pdf , 2007. Silberschatz, A., Korth, H.F. and Sudarshan, S. Database System Concepts, 6th Edition. McGraw-Hill, New York, 2010. 